\title{
Planar Kinematics Analysis of a Snake-like Robot
}

\author{
Lounis Douadi $^{1} \quad$ Davide Spinello $^{2} \quad$ Wail Gueaieb $^{1}$ \\ Hassan Sarfraz ${ }^{1}$ \\ ${ }^{1}$ University of Ottawa, School of Electrical Engineering and \\ Computer Science, 800 King Edward Ave. Ottawa Ontario \\ K1N 6N5 Canada \\ ${ }^{2}$ University of Ottawa, Department of Mechanical Engineering, \\ 161 Louis Pasteur. Ottawa Ontario K1N 6N5 Canada
}

\begin{abstract}
This paper presents the kinematics of a planar multibody vehicle which is aimed at the exploration, data collection, non-destructive testing and general autonomous navigation and operations in confined environments such as pipelines. The robot is made of several identical modules hinged by passive revolute joints. Every module is actuated with four active revolute joints and can be regarded as a parallel
\end{abstract}


mechanism on a mobile platform. The proposed kinematics allows to overcome the nonholonomic kinematic constraint which characterizes typical wheeled robots, resulting into an higher number of degrees of freedom and therefore augmented actuation inputs. Singularities in the kinematics of the modules are analytically identified. We present the dimensional synthesis of the length of the arms, obtained as the solution of an optimization problem with respect to a suitable dexterity index. Simulation results illustrate a kinematic control path following inside pipes. Critical scenarios such as $135^{\circ}$ elbows and concentric restriction are explored. Path following shows the kinematic capability of deployment of the robot for autonomous operations in pipelines, with feedback implemented by on-board sensors.

\section{Introduction}

Snake-like mobile robots distinguish themselves from their conventional wheeled counterparts by their great agility and high redundancy, which enable them to operate in environments that might be too challenging for the latter. For instance, they are more convenient to be deployed inside pipelines, ${ }^{30}$ in narrow spaces, and on the rubble of an earthquake or a major fire, to name a few. Snake-like robots proposed in the literature are typically locomoted either with passive caster wheels supporting their frames ${ }^{34,42,44}$ or with no wheels at all. ${ }^{7,26,40,41} \operatorname{In}^{38}$ a miniature snake-like robot is proposed for pipe inspection. It has the capacity of moving inside pipes with various diame- 
ters. It is part of a new family of biologically inspired robots $1,11,27,32,33,39,45,46$ as it mimics the snake's serpentine motion to propel itself inside the pipe. Robots developed for pipe inspection are Small Pipe Inspector $(\mathrm{SPI})^{20}$ and Explorer. ${ }^{36}$ To navigate inside complex pipe structures, Sigurd et al. ${ }^{14}$ conceived PIKo, a snake like robot propelled by active wheels on each module. Vertical propulsion is performed with serpentine motion. An earthworm-like mobile robot is designed in ${ }^{33}$ to navigate inside pipes using rubber bellows and friction rings to ensure friction forces with the pipe walls. A sinister exploration and pipeline inspection robot, called Kaerot-3, was developed in. ${ }^{37}$ It is a train-like robot tailored for ferromagnetic small-diameter pipes. $\mathrm{In}^{18}$ a 16-degree-of-freedom multibody mobile robot, Koryu (KR), was designed to carry out inspection tasks in a nuclear reactor. It was composed of six articulated body segments tailored for good terrain adaptability and high mobility through crawler-track wheels. Another nuclear plant inspection and maintenance robot was presented in ${ }^{13}$ but it resembled in many aspects to Koryu. Several other articulated mobile robots were developed for many other applications, like underground mine tunnels ${ }^{2,12}$ and search and rescue. $^{21,23}$

Due to the typical high redundancy of snake-like robots, various methods have been proposed to solve for their inverse kinematics. ${ }^{43}$ Such methods range from the commonly known Denavit-Hartenberg (D-H) convention, to a mathematically simpler derivative proposed in, ${ }^{35}$ to a virtual structure for orientation and position (VSOP) introduced by Liljebäk et al. ${ }^{24}$ among oth- 
ers. For instance, the simplified D-H method described in ${ }^{35}$ takes advantage of certain robot properties where only a limited number of joints can be activated at a given time.

Among wheeled snake-like robots presented in the literature, the $n$-trailer multibody vehicles made of a car or cart-like tractor towing passive cart-like trailers have been extensively studied..$^{3,6,9,12,13,21,25,28}$ A large body of research has been carried out along this direction, not only on the variants of their mechanical structures, but mostly on the control aspect. ${ }^{2,4-6,13,16,47}$ Depending on how the modules are linked together, $n$-trailer systems may be grouped into two major categories: (i) the standard $n$-trailer, for which the driven modules are directly attached to the wheel axles of the driver ones (the precedent modules), and (ii) the general $n$-trailer variant where at least one module is not attached directly to the wheel axle of its driver. The kinematics of wheeled snake robots are usually constrained by the nonholonomic property imposed by the caster wheel(s). Such a property is often used along with differential geometry to compute the net change in the robot's pose in response to the changes in the generalized joints. ${ }^{10,22,34}$

This paper is mainly concerned with wheeled snake-like robots. It studies the kinematics of a planar version of a fully-fledged robot for autonomous confined environment exploration, such as pipeline inspection. This is a part of a long-term project aiming at prototyping a commercial model of the robot. Once this part is complete, it will be extended at a later stage to cover the kinematics of the final spatial robot. Figure 1 shows the kinematic scheme 
of the proposed robot, that is inspired by Explorer ${ }^{36}$ (see figure 2 borrowed from the Carnegie Mellon web page dedicated to the robot). Modules are hinged with passive revolute joints while each module is equipped with four revolute actuators: two on the shoulder and two at the base in contact with the pipe perimeter. Such a design excels in eliminating the nonholonomic kinematic constraint which restricts the mobility of the majority of wheeled mobile robots. To the best of our knowledge, this particular parallel structure of the modules attached together to form an articulated in-pipe inspection robot has not been exploited before. This paper aims at thoroughly studying the proposed kinematic structure of this robot, and at demonstrating path following control to simulate autonomous operation of the device for exploration and inspection. This is an essential step in the direction of the development of a fully autonomous robot.

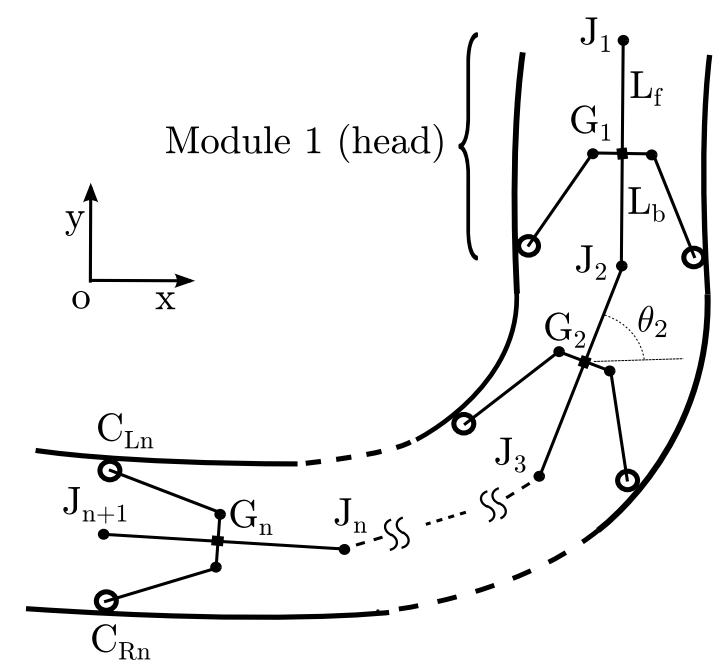

Figure 1: Schematic of a configuration of the multibody vehicle 


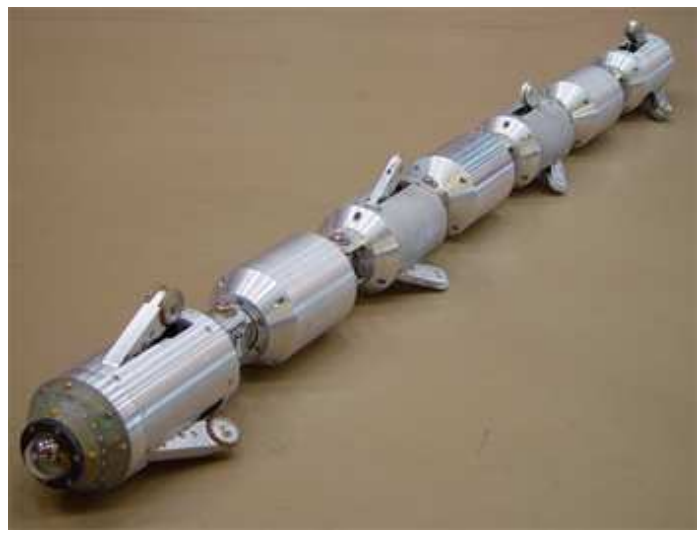

Figure 2: The articulated multibody robot Explorer (source: http://www . rec.ri.cmu.edu/projects/explorer/, active on July 2013)

The rest of the paper is organized as follows. The kinematic model of one module is detailed in section 2 along with its dimensional synthesis, qualitative analysis, control strategy and simulation results of path following in a pipe-like environment. Section 3 extends this study to the whole articulated mobile robot. Additional simulation results illustrate path following control with the articulated multibody robot. Path following control is an essential step for autonomous operation of the robot when equipped with appropriate sensors that couple navigation and estimation. Summary and conclusions are drawn in Section 4. 


\section{Modelling a Single Module: a Mobile Par- allel Mechanism}

\subsection{Description of The Mechanism}

The robot modelled here is comprised of identical modules connected by passive revolute joints. Each module is a parallel mechanism on a mobile platform $^{29}$ as represented in figure 3 . The structure supporting the main body ( $h$ high $\times W$ wide) is locomoted by two arms of length $l$, in contact with the pipeline surface using two active wheels of radius $\rho$. Each arm is attached to the body with an active revolute joint located at points $S_{r}$ and $S_{l}$ respectively, which are $(\lambda-0.5) h$ above the center of mass $G$, where $\lambda$ is a non-dimensional parameter. To ensure proper locomotion, the wheels must be in contact with the pipeline surface at points $C_{r}$ and $C_{l}$ (see figure 4). In the planar description adopted here, the pipeline surface is geometrically described by two curves that describe the walls. In practice, these curves can be obtained with the help of proximity sensors.

The module's mechanism is composed of 5 bodies: the main body, two arms and two wheels connected by four revolute joints (two at the shoulders and two at the base). Each of these bodies has 3 degrees of freedom giving a total of 15 degrees of freedom. Each revolute joint corresponds to 2 kinematic constraints and therefore a total of 8 constraints due to the joints. Additionally, the two wheels in contact with the walls are described by a 


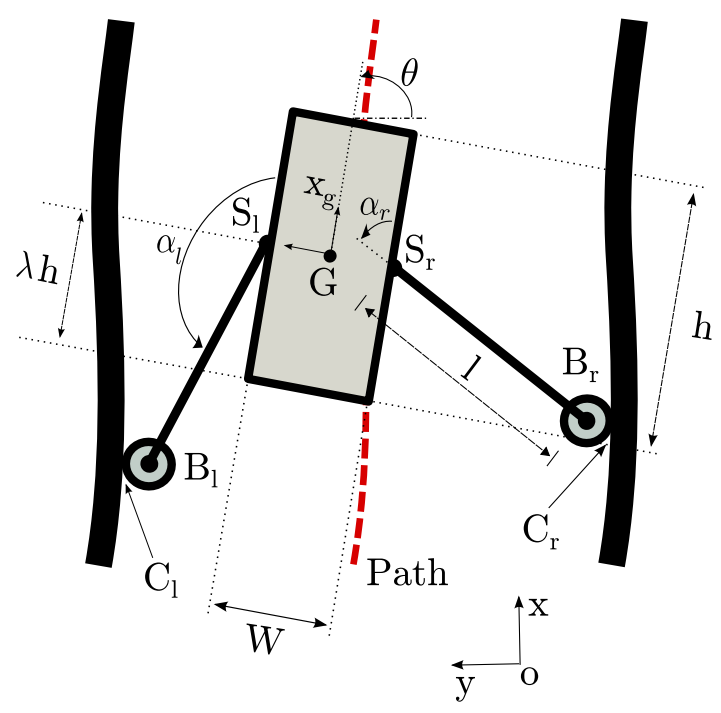

Figure 3: Sketch of a module with relevant parameter and kinematic descriptors

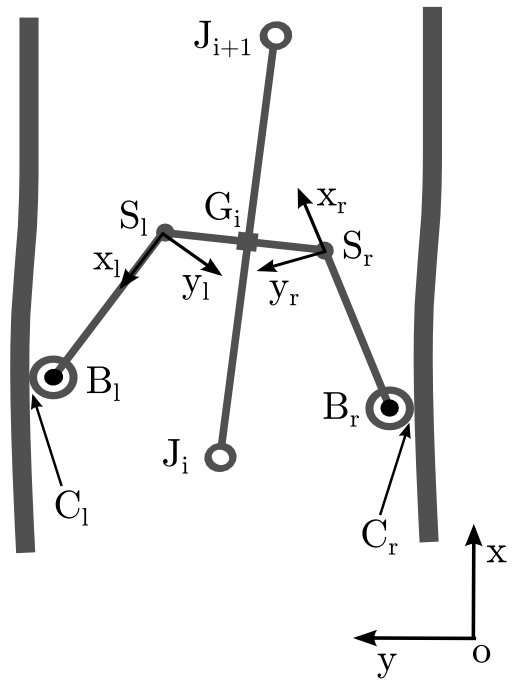

Figure 4: Kinematic scheme of one module

total of 4 constraints. This yields $3(=15-12)$ degrees of freedom which in our case correspond to the pose of the platform that is controlled by the 
shoulder joints and the active wheels. Therefore the mobility of one isolated module is 3 .

\subsection{Geometric Model}

The geometric model establishes the relation between the joints and the endeffector coordinates. We collect the three free coordinates into the vector $x=$ $\left[x_{g}, y_{g}, \theta\right]$ which describes the pose of the end effector through the coordinates $x_{g}, y_{g}$ of the center of mass and the global orientation $\theta$, see figure 3 . The joint coordinates vector $q$ contains the two joint angles $\alpha_{r}$ and $\alpha_{l}$ along with the positions of the centers of mass $B_{r}$ and $B_{l}$. In this work, the wheels are constrained to be in contact with the pipe walls; therefore the center of each wheel lies on the normal to the surface at the contact point. For a small rigid wheel, the distance between the contact point and the center is equal to the radius of the wheel $\rho$. Hence, the motion of the centers of mass of the right and left wheels are tangent to the right and left pipe walls, respectively. A diagram illustrating this for the right wheel is shown in figure 5.

Consider the Cartesian coordinate systems $F_{o}=\{O, x, y\}, F_{g}=\left\{G, x_{g}, y_{g}\right\}$, $F_{S_{l}}=\left\{S_{l}, x_{l}, y_{l}\right\}$, and $F_{S_{r}}=\left\{S_{r}, x_{r}, y_{r}\right\}$, with origins $o, G, S_{l}$ and $S_{r}$, respectively (see figures 3 and 4 ). To express a quantity in a specific coordinate system we adopt the notation $(\cdot)_{/ x}$, where $x \in\{o, g, l, r\}$. Let $\Pi_{r}\left(\tau_{r}\right)=$ $\left[\Pi_{r}^{x}\left(\tau_{r}\right) \quad \Pi_{r}^{y}\left(\tau_{r}\right)\right]^{T}$ be the parametric curve describing the right pipe wall as a

function of the arc length parameter $\tau_{r}$, and $N_{r}\left(\tau_{r}\right)=\left[\begin{array}{lll}N_{r}^{x}\left(\tau_{r}\right) & N_{r}^{y}\left(\tau_{r}\right)\end{array}\right]^{T}$ be the normal to the right wall at point $C_{r}$, all expressed in the global frame. 


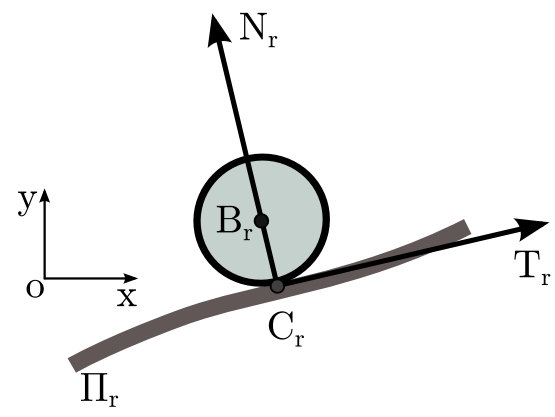

Figure 5: Illustration of the kinematic constraint describing the contact of the right wheel with the pipe wall

Based on the contact kinematic constraint described above, the coordinates of the right wheel center can be expressed as a function of the arc length parameter $\tau_{r}$

$$
\begin{gathered}
O B_{r_{/ o}}\left(\tau_{r}\right)=\Pi_{r}\left(\tau_{r}\right)+\rho N_{r}\left(\tau_{r}\right) \\
O B_{l / o}\left(\tau_{l}\right)=\Pi_{l}\left(\tau_{l}\right)+\rho N_{l}\left(\tau_{l}\right)
\end{gathered}
$$

where we have extended the notation to the left wheel through the subscript $l$. Therefore, the positions of the wheel centers $B_{r}$ and $B_{l}$ are determined by the arc length parameters $\tau_{r}$ and $\tau_{l}$, respectively, through equations (1), and the joints coordinates vector is given by $q=\left[\alpha_{r}, \alpha_{l}, \tau_{r}, \tau_{l}\right]$.

Let $R(\cdot)$ denote a 2-D rotation matrix defined by

$$
R(\cdot)=\left[\begin{array}{cc}
\cos (\cdot) & -\sin (\cdot) \\
\sin (\cdot) & \cos (\cdot)
\end{array}\right],
$$


As graphically illustrated in figures 3 and 4 , the vector $O G_{/ o}=\left[x_{g}, y_{g}\right]$ can be equivalently expressed in the global reference frame by following paths through the right and through the left sides of the mechanism:

$$
\begin{aligned}
& O G_{/ o}=O B_{r_{/ o}}-S_{r} B_{r / o}-G S_{r / o} \\
& O G_{/ o}=O B_{l_{/ o}}-S_{l} B_{l / o}-G S_{l / o}
\end{aligned}
$$

We characterize different vectors in their local frames as

$$
\begin{array}{rlrl}
S_{l} B_{l / l} & =\left[\begin{array}{ll}
l & 0
\end{array}\right]^{T} & S_{r} B_{r / r} & =\left[\begin{array}{ll}
-l & 0
\end{array}\right]^{T} \\
G S_{l / g} & =\left[\begin{array}{lll}
H & W / 2
\end{array}\right]^{T} & G S_{r / g} & =\left[\begin{array}{ll}
H & -W / 2
\end{array}\right]^{T} \\
O B_{l / o} & =\left[\begin{array}{lll}
x_{l} & y_{l}
\end{array}\right]^{T} & O B_{r / o} & =\left[\begin{array}{ll}
x_{r} & y_{r}
\end{array}\right]^{T}
\end{array}
$$

where $H=h(\lambda-0.5)$. Given

$$
\begin{gathered}
S_{r} B_{r / o}=R(\theta) R\left(\alpha_{r}\right) S_{r} B_{r / r}, \quad G S_{r / o}=R(\theta) G S_{r / g} \\
S_{l} B_{l / o}=R(\theta) R\left(\alpha_{l}\right) S_{l} B_{l / l}, \quad G S_{l / o}=R(\theta) G S_{l / g}
\end{gathered}
$$

substitution in (2) yields

$$
\begin{aligned}
& x_{g}=x_{r}\left(\tau_{r}\right)-H \cos \theta-\frac{W}{2} \sin \theta+l \cos \left(\theta+\alpha_{r}\right) \\
& y_{g}=y_{r}\left(\tau_{r}\right)-H \sin \theta+\frac{W}{2} \cos \theta+l \sin \left(\theta+\alpha_{r}\right) \\
& x_{g}=x_{l}\left(\tau_{l}\right)-H \cos \theta+\frac{W}{2} \sin \theta-l \cos \left(\theta+\alpha_{l}\right)
\end{aligned}
$$




$$
y_{g}=y_{l}\left(\tau_{l}\right)-H \sin \theta-\frac{W}{2} \cos \theta-l \sin \left(\theta+\alpha_{l}\right)
$$

The rearrangement of equations having the same left hand side gives the closure equations of the mechanism ${ }^{29}$

$$
\begin{gathered}
l \cos \left(\theta+\alpha_{r}\right)=x_{l}\left(\tau_{r}\right)-x_{r}\left(\tau_{r}\right)-l \cos \left(\theta+\alpha_{l}\right)+W \sin \theta \\
l \sin \left(\theta+\alpha_{r}\right)=y_{l}\left(\tau_{l}\right)-y_{r}\left(\tau_{l}\right)-l \sin \left(\theta+\alpha_{l}\right)-W \cos \theta
\end{gathered}
$$

Solving the system (3) for $x$ provides the forward geometric model of the mechanism, while solving for $q$ gives the inverse geometric model.

\subsection{Differential Kinematics}

The differential kinematics is the relation between the joints and end-effector's velocities in the tangent space through appropriate Jacobians. Time differentiating (3) yields

$$
\begin{gathered}
\dot{x}_{g}+d_{r_{1}} \dot{\theta}=\dot{x}_{r}\left(\tau_{r}\right)+d_{r_{2}} \dot{\alpha}_{r} \\
\dot{y}_{g}+e_{r_{1}} \dot{\theta}=\dot{y}_{r}\left(\tau_{r}\right)+e_{r_{2}} \dot{\alpha}_{r} \\
\dot{x}_{g}+d_{l_{1}} \dot{\theta}=\dot{x}_{l}\left(\tau_{l}\right)+d_{l_{2}} \dot{\alpha}_{l} \\
\dot{y}_{g}+e_{l_{1}} \dot{\theta}=\dot{y}_{l}\left(\tau_{l}\right)+e_{l_{2}} \dot{\alpha}_{l}
\end{gathered}
$$


where $d_{r_{i}}, e_{r_{i}}, d_{l_{i}}$ and $e_{l_{i}},(i=1,2)$ are configuration dependent parameters given by

$$
\begin{array}{r}
d_{r_{1}}=\frac{1}{2} W \cos \theta+l \sin \left(\theta+\alpha_{r}\right)-H \sin \theta \\
d_{r_{2}}=-l \sin \left(\theta+\alpha_{r}\right) \\
e_{r_{1}}=\frac{1}{2} W \sin \theta-l \cos \left(\theta+\alpha_{r}\right)+H \cos \theta \\
e_{r_{2}}=l \cos \left(\theta+\alpha_{r}\right) \\
d_{l_{1}}=-\frac{1}{2} W \cos \theta-l \sin \left(\theta+\alpha_{l}\right)-H \sin \theta \\
d_{l_{2}}=l \sin \left(\theta+\alpha_{l}\right) \\
e_{l_{1}}=-\frac{1}{2} W \sin \theta+l \cos \left(\theta+\alpha_{l}\right)+H \cos \theta \\
e_{l_{2}}=-l \cos \left(\theta+\alpha_{l}\right)
\end{array}
$$

In the tangent space we describe the contact of the wheels on the walls through rolling without slipping constraints. Thus,

$$
\begin{gathered}
\omega_{r} \times \underline{r}_{r}+\underline{v}_{r}=0 \\
\omega_{l} \times \underline{r}_{l}+\underline{v}_{l}=0
\end{gathered}
$$

where $\underline{r}_{r}=\left[r_{r}^{T}, 0\right], \underline{r}_{l}=\left[r_{l}^{T}, 0\right], \underline{v}_{r}=\left[v_{r}^{T}, 0\right]$ and $\underline{v}_{l}=\left[v_{l}^{T}, 0\right]$ are vectors in $\mathbb{R}^{3}$, with $r_{r}=-\rho N_{r}$ and $r_{l}=\rho N_{l}$ being the radii vectors of the right and left wheels, consistently with the contact constraints (1). The vectors $v_{r}=\left[\dot{x}_{r}\left(\tau_{r}\right), \dot{y}_{r}\left(\tau_{r}\right)\right]$ and $v_{l}=\left[\dot{x}_{l}\left(\tau_{l}\right), \dot{y}_{l}\left(\tau_{l}\right)\right]$ are the linear velocities of the 
wheel centers, and $\omega_{r}=\left[0,0, \dot{\theta}_{r}\right]$ and $\omega_{l}=\left[0,0, \dot{\theta}_{l}\right]$ are the angular velocity vectors of the right and left wheels, respectively. In the Cartesian inertial frame, we obtain

$$
\begin{aligned}
\dot{x}_{r}\left(\tau_{r}\right)+\rho N_{r}^{y} \dot{\theta}_{r} & =0 \\
\dot{y}_{r}\left(\tau_{r}\right)-\rho N_{r}^{x} \dot{\theta}_{r} & =0 \\
\dot{x}_{l}\left(\tau_{l}\right)+\rho N_{l}^{y} \dot{\theta}_{l} & =0 \\
\dot{y}_{l}\left(\tau_{l}\right)-\rho N_{l}^{x} \dot{\theta}_{l} & =0
\end{aligned}
$$

Equations (9) establish a relation between the arc length rates, $\dot{\tau}_{r}$ and $\dot{\tau}_{l}$, and the wheels angular velocities $\dot{\theta}_{r}$ and $\dot{\theta}_{l}$. Therefore, we can introduce the joint velocity vector $\dot{q}=\left[\dot{\alpha}_{r}, \dot{\alpha}_{l}, \dot{\theta}_{r}, \dot{\theta}_{l}\right]$ that conveniently include the angular velocities. By collecting (6) and (9) we can write the velocity kinematics in matrix form as

$$
J_{x} \dot{x}=J_{q} \dot{q}
$$

where $J_{x} \in \mathbb{R}^{4 \times 3}$ and $J_{q} \in \mathbb{R}^{4 \times 4}$ are, respectively, the parallel and the serial Jacobians of the mechanism

$$
J_{q}=\left[\begin{array}{cccc}
d_{r_{2}} & 0 & -\rho N_{r}^{y} & 0 \\
e_{r_{2}} & 0 & \rho N_{r}^{x} & 0 \\
0 & d_{l_{2}} & 0 & -\rho N_{l}^{y} \\
0 & e_{l_{2}} & 0 & \rho N_{l}^{x}
\end{array}\right] \quad J_{x}=\left[\begin{array}{ccc}
1 & 0 & d_{r_{1}} \\
0 & 1 & e_{r_{1}} \\
1 & 0 & d_{l_{1}} \\
0 & 1 & e_{l_{1}}
\end{array}\right] .
$$


If $J_{x}$ is full rank the two can be combined to one Jacobian $J$ to obtain

$$
\dot{x}=J \dot{q}
$$

where

$$
J=\left(J_{x}^{T} J_{x}\right)^{-1} J_{x}^{T} J_{q}
$$

\subsection{Singular Positions}

Singularities occur when one or both Jacobians, $J_{x}$ and $J_{q}$, become singular. ${ }^{17}$ A parallel singularity occurs when the parallel Jacobian $J_{x}$ takes a singular value. This means that the robot is at a configuration where tangent motions of the end-effector are admissible while the joints are locked. A serial singularity occurs when the serial Jacobian $J_{q}$ is singular, corresponding to configurations where the robot loses one or more degrees of freedom. ${ }^{29}$ For the robot studied here, a serial singularity occurs when $\operatorname{det} J_{q}=0$. This implies

$$
N_{l}^{x} \sin \left(\theta+\alpha_{l}\right)=N_{l}^{y} \cos \left(\theta+\alpha_{l}\right) \quad \text { or } \quad N_{r}^{x} \sin \left(\theta+\alpha_{r}\right)=N_{r}^{y} \cos \left(\theta+\alpha_{r}\right) .
$$

These conditions correspond to configurations where one or both arms of the robot are normal to the corresponding pipe wall. Figure 6 illustrates two examples of such cases. The singularity position depicted in figure 6(a) 
occurs when each arm is perpendicular to the surface it is in contact with. It can be easily avoided by having the mechanism's span to be larger than the pipe diameter. Nevertheless, serial singular configurations as in figure 6(b) are still physically possible. Avoiding them can be encoded as part of the controller's objectives.

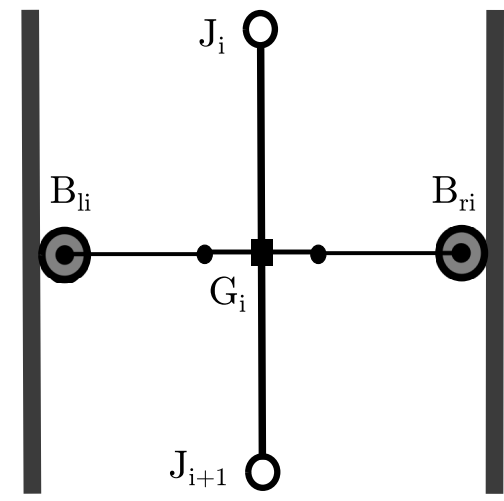

(a)

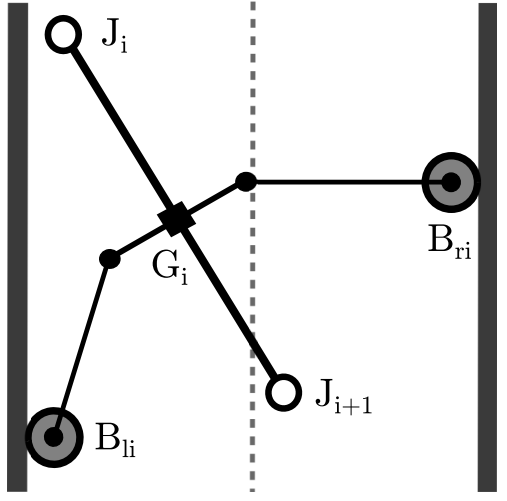

(b)

Figure 6: Two serial singularities: (a) both arms are normal to the pipe walls, (b) only one arm is normal to the wall

The parallel singularities occur when the parallel Jacobian $J_{x}$ is singular, or if it is not of full rank for a non-square Jacobian. We study the rank of $J_{x}$ through the eigenvalues of matrix $J_{x}^{T} J_{x}$ based on the fact that the singular values of $J_{x}$ are the square roots of the eigenvalues of $J_{x}^{T} J_{x}$, and by using the fact that the rank of $J_{x}$ equals the number of its nonzero singular values. ${ }^{8}$ If $\operatorname{det}\left(J_{x}^{T} J_{x}\right) \neq 0$, then all its eigenvalues are non-zero, and therefore all the singular values of $J_{x}$ are non-zero, which implies that $J_{x}$ is full rank. The 
determinant of $J_{x}^{T} J_{x}$ may be expressed as

$$
\operatorname{det}\left(J_{x}^{T} J_{x}\right)=W^{2}+2 l^{2}\left(1+\cos \left(\alpha_{r}-\alpha_{l}\right)\right)+2 W l\left(\sin \alpha_{r}+\sin \alpha_{l}\right) .
$$

This determinant vanishes when $\alpha_{r}-\alpha_{l}=\pi$ and $W=0$. Figure 7 depicts a configuration corresponding to those conditions. We assume that the module width $W$ is nonzero. Therefore, by setting the constraints $0<\alpha_{r}<\frac{\pi}{2}$ and $\frac{\pi}{2}<\alpha_{r}<\pi$ on the shoulder joints, the determinant never vanishes, and parallel singularities cannot occur.
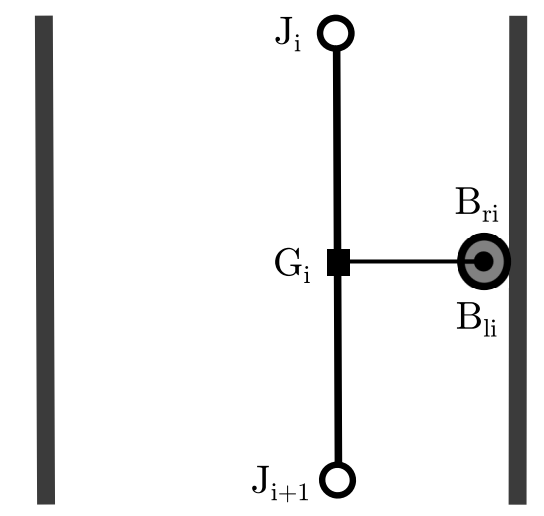

Figure 7: Right wheel contact with the pipe wall

\subsection{Dimensional Synthesis : Optimal Design of The Arms}

We have established that in order to avoid serial singularities the span of the module must be larger than the pipe diameter. Since parallel singularities are not admitted given the constraints on the joints span, for the rest of this 
section we will use the term singularity as a synonymous of serial singularity. Here we illustrate a procedure to find the optimal arm's length $l$ with the objective of maximizing the singularity-free workspace, for a scenario that involves crossing a $135^{\circ}$ elbow. This typically constitutes a difficult task from an operational point of view (see figure 8). The optimization must take into account collision avoidance of the module's main body and of the arms with the pipe walls.

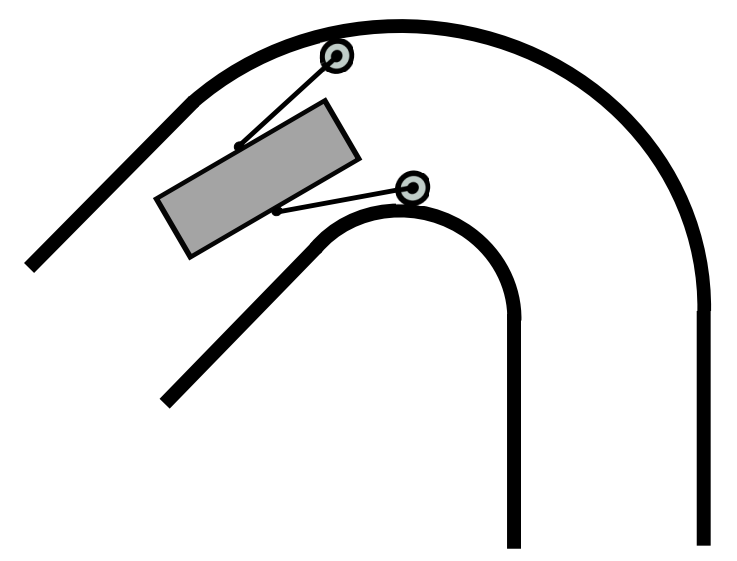

Figure 8: Illustration of the module in a $135^{\circ}$ elbow

The optimization procedure is based on the dexterity index ${ }^{19}$ defined by

$$
\delta=\frac{\Gamma_{s f}}{\Gamma_{r e f}} .
$$

where $\Gamma_{s f}$ denotes the intersection of singularity-free and collision-free workspaces, while $\Gamma_{r e f}$ denotes the reference workspace. The latter is computed numerically by sampling the surface area inside the pipe along the $x$ an $y$ directions with a step size of $1 \cdot \mu$, for a unity length $\mu . \Gamma_{s f}$ is also computed numerically 
by pinning the module's center of gravity $G$ on every sampled point in $\Gamma_{r e f}$ and spanning its orientation angle $\theta$ in $\left[\theta_{\nu}-\frac{\pi}{4}, \theta_{\nu}+\frac{\pi}{4}\right]$ with an angular step of $\frac{10 \pi}{180} \mathrm{rad}$, where $\theta_{\nu}$ is the orientation of the tangent to the center line of the pipe at every position of the orthogonal projection of $G$ on this line. This process is repeated for different values of the arm length $l$ while fixing the rest of the parameters to $W=10 \mu, h=35 \mu$, and the pipe width $W_{p}=42 \mu$. The optimal length is the one maximizing the dexterity index.

Numerical simulations were carried out with a unity length $\mu=1 \mathrm{~cm}$. Figure 9 shows the dexterity index against the orientation angle $\theta$ for different arm lengths. It can be seen that the optimal dexterity is attained for an arm length $l=24 \mathrm{~cm}$. The figure also shows that the dexterity index in the vicinity of the orientation of the tangent to the path is always close the maximum dexterity value. The plots are not symmetric due to the collision avoidance constraints which shrink the workspace asymmetrically along the elbow. Figure 10 shows the average dexterity over the orientation angle span versus the arm length. Once again, the optimal dexterity is reached when $l=24 \mathrm{~cm}$, which is consistent with the previous result.

The reciprocal of the condition number of the serial Jacobian provides a proximity measure to the robot's singular configurations. Let $0<\Phi<1$ denote this proximity measure, i.e.,

$$
\Phi=\frac{1}{\left\|J_{q}\right\|\left\|J_{q}^{-1}\right\|} .
$$




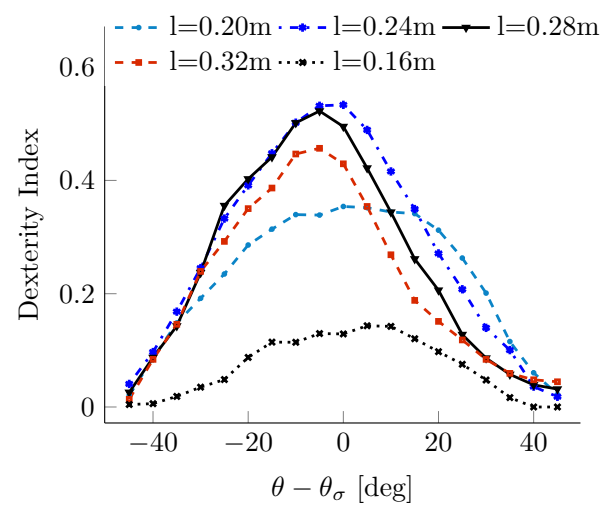

Figure 9: Dexterity index $\delta$ versus relative orientation $\theta-\theta_{\nu} \in[-\pi / 2, \pi / 4]$, for different arm lengths $l$

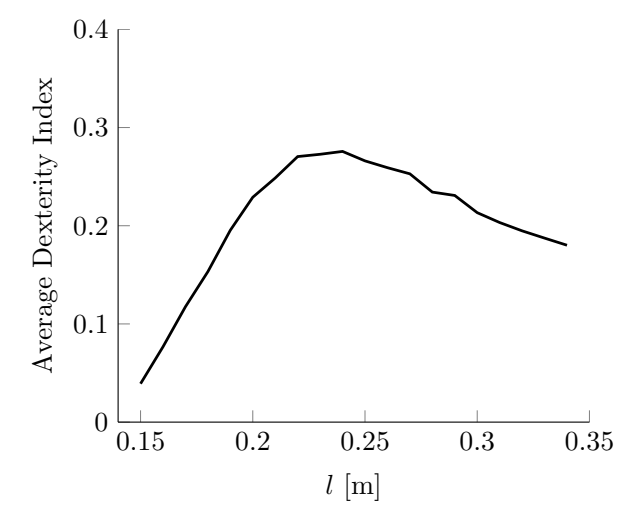

Figure 10: Average dexterity index over the full span $\left[\theta_{\nu}-\pi / 4, \theta_{\nu}+\pi / 4\right]$ of the global orientations of the module, for different values of $l$

The closer $\Phi$ is to zero the closer the robot's configuration is to singularity; the closer it is to 1 the closer the Jacobian is to isotropy. Figures 11 and 12 show the variations of $\Phi$ inside the pipeline for two different orientations of the robot corresponding to $\theta=\theta_{\nu}$ and $\theta=\theta_{\nu}-\frac{\pi}{6}$, respectively. The variation is visually illustrated with a color-gradient map ranging from red, for $\Phi=0$, to green, for $\Phi=1$. The figures show that the highest values of $\Phi$ 
occur around the center line of the pipe and it decreases as the robot moves away toward the edge. It is important to notice the smooth variation in $\Phi$ across the workspace in both cases. There are no isolated islands of green regions which means that we obtain a non-vanishing workspace, which is a very important feature of the proposed robot.

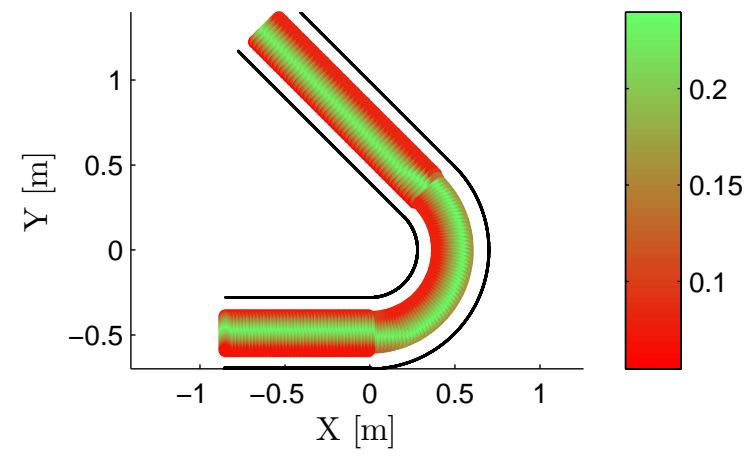

Figure 11: Workspace illustration for orientation $\theta=\theta_{\nu}$

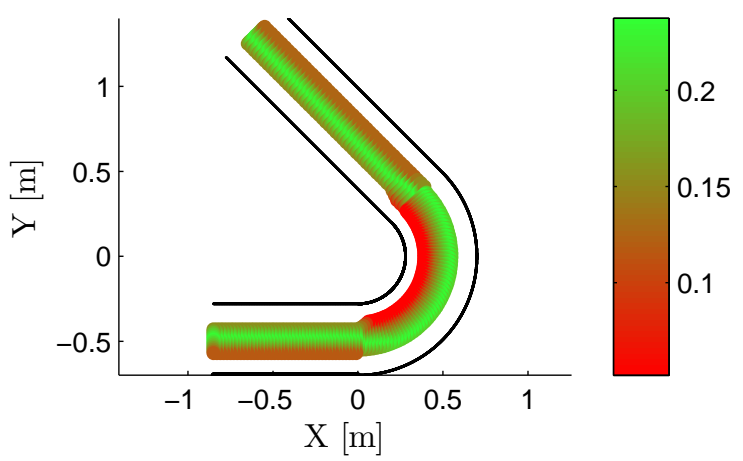

Figure 12: Workspace illustration for $\theta=\theta_{\nu}-\frac{\pi}{6}$ 


\subsection{Forward Motion Control: A Show Case}

To illustrate the practicality of the robot's kinematics we hereby provide a simulation example on a simple control scheme driving a single module inside a pipeline. Note that the purpose of this study is not to devise the best controller for the module, but rather to have some understanding of how adequate the proposed module structure and the afore-derived kinematic model can be for navigating inside a confined environment. The control objective in this example is to make the module's center of mass $G$ slide along the central axis of the pipe while keeping its orientation tangent to it. In practice, the position of the pipe center point can be computed by measuring the distance and curvature of each side of the pipe through an array of proximity sensors mounted on both sides of the mechanism.

The pose of the module is expressed with respect to a mobile Frenet frame, $F_{/ p}=\left\{P, T^{P}, N^{P}\right\}$, attached to the path. ${ }^{15,25}$ The origin $P=\left[x_{p}(\tau), y_{p}(\tau)\right]$ is the orthogonal projection of $G$ on the path, as shown in figure 13, and $\tau$ is the arc length parameter on the path. The axes $T^{P}$ and $N^{P}$ are the tangent and the normal to the path at point $P$, respectively. The module's desired pose $x^{d}$ is expressed as $x^{d}=\left[x_{p}, y_{p}, \theta_{\nu}\right]$, where $\theta_{\nu}$ is the orientation angle of $T^{P}$ which represents the module's desired orientation angle. Recall that the module's actual pose was previously defined as $x=\left[x_{g}, y_{g}, \theta\right]$. In this context, the module's tracking error $e$ is defined as $e=\|G-P\|$. The orientation

error $\tilde{\theta}$ is defined by $\tilde{\theta}=\theta_{\nu}-\theta$. In analogy to parallel manipulators, the path following control of this module can be regarded as a parallel manipulator 
whose end-effector position $G$ is controlled to track a moving virtual point $P$ with a desired orientation.

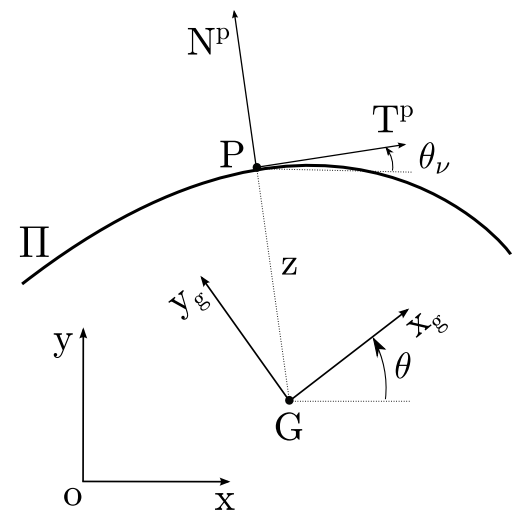

Figure 13: Frenet frames for path following control

A simple proportional controller is adopted to generate the module's operational velocity $\dot{x}$ to be proportional to the pose error $\tilde{x}=x^{d}-x$. The module's dynamics is simplified here as a point mass of a negligible inertia such that $\dot{x}=u$ with $u$ being the control effort. The path following problem can be formulated as tracking the desired evolution of the position and orientation of a point on the path, and reject the disturbance represented by the curvature of the path. ${ }^{4}$ Given the elbow with constant curvature considered in our simulation a proportional controller is sufficient to asymptotically reject the corresponding step disturbance. Therefore the control effort is formulated $\mathrm{as}^{31}$

$$
u=K \tilde{x}+v
$$


The matrix $K=\operatorname{diag}\left(K_{x}, K_{y}, K_{\theta}\right) \in \mathbb{R}^{3 \times 3}$ is a diagonal positive definite gain matrix, $v=V\left[\cos \theta_{\nu}(\tau), \sin \theta_{\nu}(\tau), \kappa(\tau)\right], V$ is a non-negative scalar, and $\kappa(\tau)=\frac{d \theta_{\nu}}{d s}$ is the curvature of the path at point $P(\tau)$. Taking $V$ as $\frac{d s}{d t}$, where $d s$ is an infinitesimal displacement of $P$, leads to $V \kappa(\tau)=\frac{d \theta_{\nu}}{d t}=\dot{\theta}_{\nu}$. Therefore, the vector $v$ is in fact the desired velocity, i.e., $v=\dot{x}^{d}$. Hence, (12) can be rewritten as $u=\dot{x}=K \tilde{x}+\dot{x}^{d}$, and so $K \tilde{x}+\dot{\tilde{x}}=0$. This is a well known form of asymptotically stable systems for a positive definite matrix $K$ for which the pose error and its velocity asymptotically converge to zero. As a result, $\lim _{t \rightarrow \infty} \tilde{x}=\lim _{t \rightarrow \infty} \dot{\tilde{x}}=0$.

Simulations were conducted to test the behaviour of a single module under the command of this controller to make it track the center line of a pipe with a $135^{\circ}$ elbow with outer radius of $70 \mathrm{~cm}$. The geometry of the module is determined by the parameters $l=24 \mathrm{~cm}, h=35 \mathrm{~cm}, \lambda=0.5, w=$ $10 \mathrm{~cm}$, and $\rho=3 \mathrm{~cm}$. This set of parameters ensures collision avoidance with the walls for the set of trajectories generated in this simulation. Time derivatives are approximated by forward finite difference approximations with step 0.005 sec. The results are revealed in Figures 14 and 15. One can notice two saddle points in the orientation error plot. This is due to two changes in the path curvature corresponding to the points when the module enters and leaves the elbow. Figure 15 shows three snapshots of the module while navigating through the pipe (initial, intermediate and final positions). It is worth noticing how the tracking error evolved from a non-zero value initially to practically nil. 


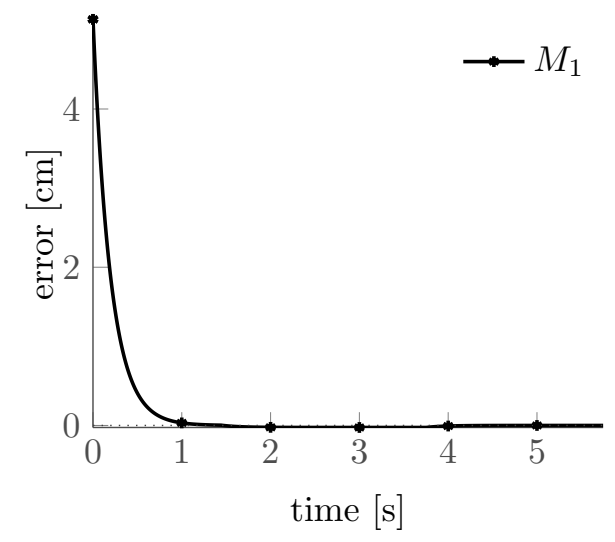

(a) tracking error

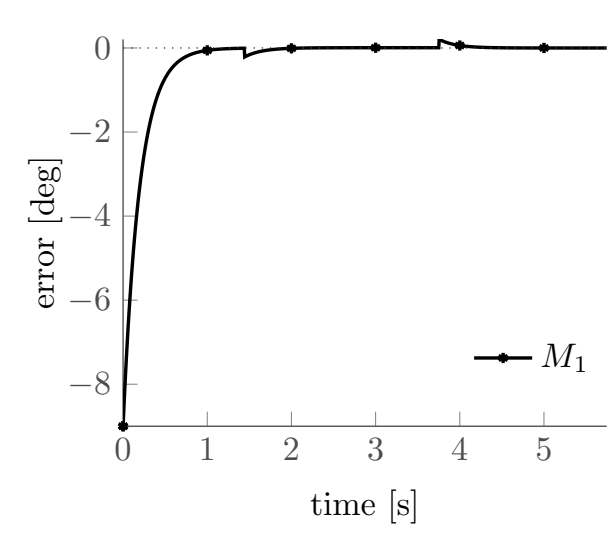

(b) orientation error

Figure 14: Position and orientation errors in an elbow with one module

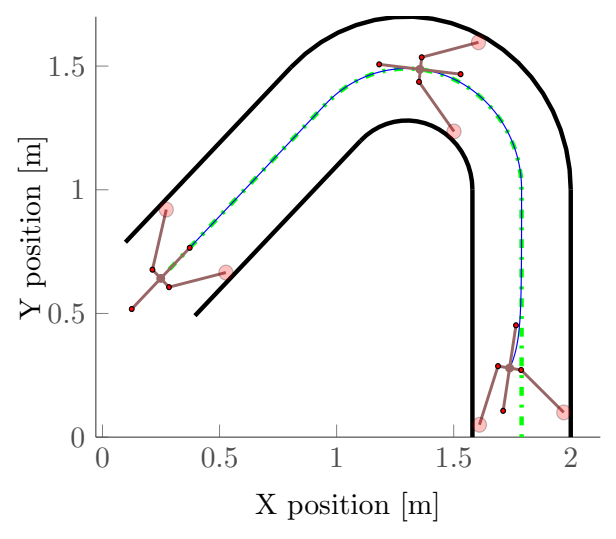

Figure 15: Module's snapshots in an elbow

\section{Articulated Vehicle}

\subsection{Kinematic Model}

In this section we address the kinematics of the full articulated vehicle obtained by connecting the modules studied above. Hitching two modules together by a revolute joint introduces two kinematic constraints. Given that 
each module has 3 independent scalar states, for a vehicle made of $n$ connected modules with $n-1$ revolute joints the number of degrees of freedom is $3 n-2(n-1)=n+2$. The configuration of the full robot is defined by the operational and the joint coordinate vectors $\psi=\left[x_{1}, \ldots, x_{n}\right]$ and $\phi=\left[q_{1}, \ldots, q_{n}\right]$, respectively, such that

$$
x_{i}=\left[x_{g_{i}}, y_{g_{i}}, \theta_{i}\right]
$$

and

$$
q_{i}=\left[\alpha_{r_{i}}, \alpha_{l_{i}}, \theta_{r_{i}}, \theta_{l_{i}}\right]
$$

are respectively the operational and the joint coordinates of module $M_{i}$, $i \in\{1, \ldots, n\}$. Extending (11) to the articulated robot and adding the proper subscript for each module, we get the following matrix form expression

$$
A=\left(\begin{array}{cccc}
\dot{\psi}=A \dot{\phi} & \\
J_{1} & & & 0_{3 \times 4(n-1)} \\
0_{3 \times 4} & J_{2} & & 0_{3 \times 4(n-2)} \\
\vdots & \vdots & \ddots & \vdots \\
0_{3 \times 4(n-1)} & & & J_{n}
\end{array}\right) .
$$


The rigid body geometric constraints relating two consecutive modules $M_{i}$ and $M_{i-1}$ are

$$
\begin{gathered}
x_{g_{i}}=x_{g_{i-1}}-L_{b} \cos \theta_{i-1}-L_{f} \cos \theta_{i} \\
y_{g_{i}}=y_{g_{i-1}}-L_{b} \sin \theta_{i-1}-L_{f} \sin \theta_{i}
\end{gathered}
$$

where $L_{b}$ and $L_{f}$ are the lengths of segments $G_{i} J_{i+1}$ and $G_{i} J_{i}$ respectively (see figure 1) which are equal in our case. Time deriving the equations above we obtain the velocity constraints

$$
\begin{aligned}
& \dot{x}_{g_{i}}=\dot{x}_{g_{i-1}}+L_{b} \dot{\theta}_{i-1} \sin \theta_{i-1}+L_{f} \dot{\theta}_{i} \sin \theta_{i} \\
& \dot{y}_{g_{i}}=\dot{y}_{g_{i-1}}-L_{b} \dot{\theta}_{i-1} \cos \theta_{i-1}-L_{f} \dot{\theta}_{i} \cos \theta_{i}
\end{aligned}
$$

With this setup, the robot's pose velocity $\dot{\psi}$ may be computed from (13) subject to constraints (17). Note that the joint coordinates $\phi$ and/or their velocities $\dot{\phi}$ can be readily measured through sensors attached to the four activated joints of each module.

\subsection{Motion Control of the Articulated Vehicle}

We illustrate the behavior of the articulated robot by exciting it with a simple controller as we did earlier for a single module. Here, we control the pose of the leading module (head) and the orientation angles of the trailing modules. Let $\xi=\left[x_{1}^{T}, \theta_{2}, \ldots, \theta_{n}\right]$ be the state vector comprised of the independent 
states. Then the controller is to track $\xi^{d}=\left[x_{p_{1}}, y_{p_{1}}, \theta_{\nu 1}, \theta_{\nu 2}, \ldots, \theta_{\nu n}\right]^{T}$, which is an extended version of $x^{d}$ in section 2.6. Generalizing the $\mathrm{P}$ controller discussed earlier to the whole robot, we get a control signal $u$ given by

$$
u=K \tilde{\xi}+v
$$

with $\tilde{\xi}=\xi^{d}-\xi, K=\operatorname{diag}\left(K_{x}, K_{y}, K_{\theta_{1}}, \ldots, K_{\theta_{n}}\right)$ is a diagonal positive definite matrix, $v=V\left[v_{1}, \ldots, v_{n}\right], v_{i}=\left[\cos \theta_{\nu_{i}}, \sin \theta_{\nu_{i}}, \kappa_{i}\right]$, and $V$ being a non-negative scalar. Note that $\theta_{\nu_{i}}$ and $\kappa_{i}$ are the orientation of the Frenet frame attached to module $M_{i}$, and the curvature of the path at the arc length position of $M_{i}$, respectively. Simulations were run on a robot of 5 modules with two different scenarios, with time derivatives approximated by forward finite differences with time step $0.0125 \mathrm{sec}$. In the first scenario, the robot is set to cross a straight $6 \mathrm{~m}$-long pipe with a varying diameter. It extends for $2.5 \mathrm{~m}$ with a diameter of $42 \mathrm{~cm}$, then the diameter reduces to $34 \mathrm{~cm}$ for $1 \mathrm{~m}$ before it enlarges to $39 \mathrm{~cm}$. The geometry of each module is determined by the set of parameters $l=24 \mathrm{~cm}, h=35 \mathrm{~cm}, \lambda=0.5, w=10 \mathrm{~cm}$, and $\rho=3 \mathrm{~cm}$.. The results of this simulation are shown in figures 16 and 17 . All modules converge to and follow the central axis of the pipe, and therefore the tracking error converges to zero.

In the second scenario the robot is set to track the center line of a pipe with varying (piecewise constant) curvature. Specifically, the portion of the pipe is made of two straight segments joined by a $135^{\circ}$ elbow. The results 


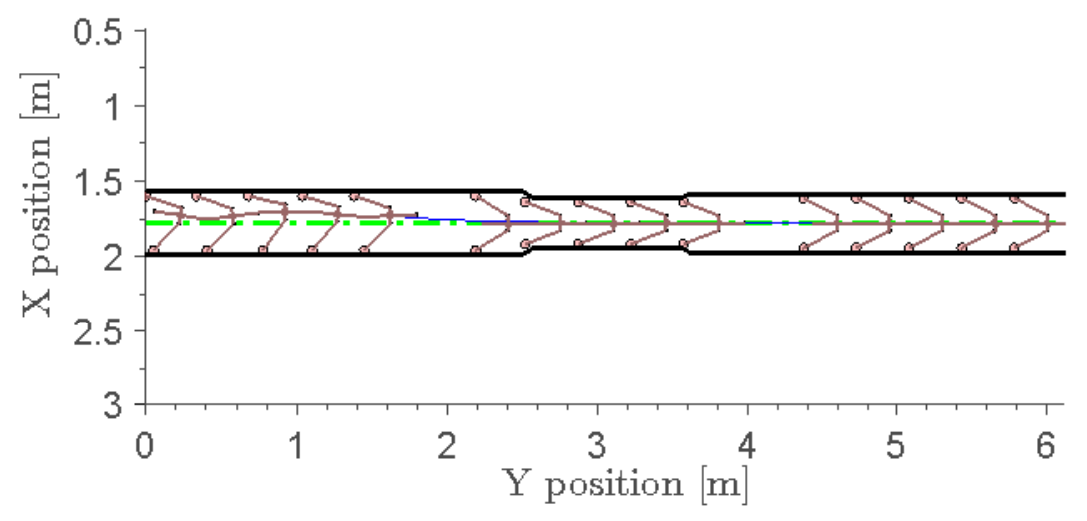

Figure 16: Snapshots of a 5-module robot in a pipe of varying diameter

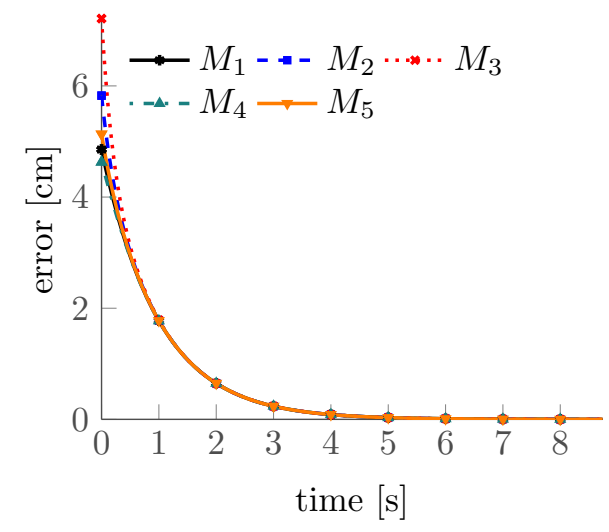

(a) tracking error

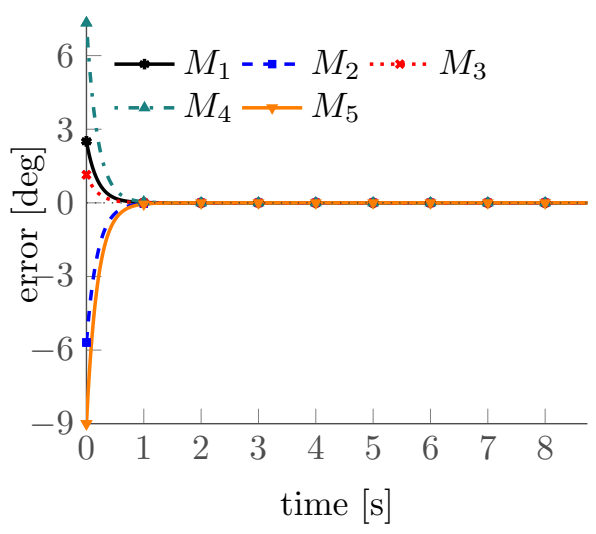

(b) orientation error

Figure 17: Position and orientation errors of a 5-module robot in a pipe of varying diameter

are shown in figures 18 and 19. As expected, the orientation errors asymptotically vanish for all modules. However, the relative position error with respect to the path propagates from the head and it gets amplified between two subsequent modules. This is consistent with the fact that, except for the head, the relative position of the center of mass with respect to the path is 
not controlled, and therefore there is no self regulation mechanism to reject the disturbances in the form of non-zero curvature of the elbow. ${ }^{4}$ The amplification can be kinematically explained by the fact that a change of curvature causes the lateral shift of the entire trailer (when module $M_{i}$ enters into the elbow modules $M_{i+1}$ to $M_{n}$ are simultaneously pushed to the right of the path as a consequence of the motion of joint $J_{i+1}$ ). This coupling implies that the $i$-th module experiences $i$ disturbances for each change of curvature, and therefore the cumulative effect resulting in the amplification and peaks in the position error.

The disturbance increases with the curvature. ${ }^{4}$ This is illustrated by two additional simulations in which we consider, respectively, $90^{\circ}$ and $270^{\circ}$ elbows. The first case is shown in figures 20 and 21, respectively with snapshots of the robot inside a pipe with $90^{\circ}$ elbow and inside a pipe with $270^{\circ}$ elbow. The $90^{\circ}$ elbow has the same radius as the one in figure $18(70 \mathrm{~cm})$, whereas the $270^{\circ}$ elbow has the larger radius $1 \mathrm{~m}$. Figures 22 and 23 depict the distance and orientation errors for both simulations. By comparing these results with the results in figure 19 a smaller error peak magnitude is revealed for the larger radius elbow, consistently with the theoretical characterization of the curvature as disturbance.

For applications in which the position error with respect to the path is an important performance index (for example, if the payload of the modules is a sensor with performance affected by the proximity to the pipe axis), different control schemes that regulate the position error should be considered. ${ }^{5}$ 


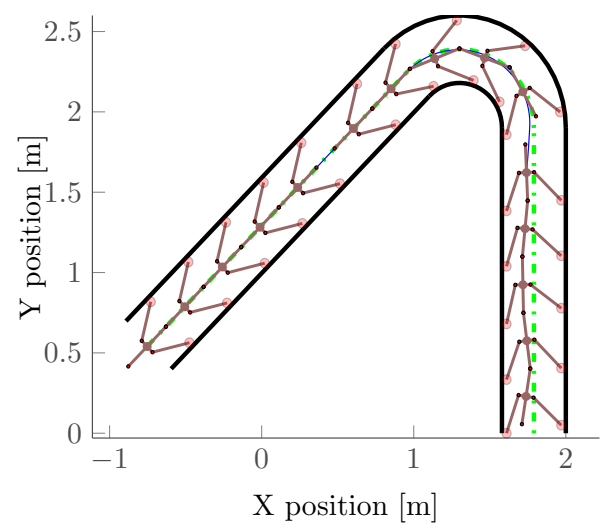

Figure 18: Snapshots of a 5-module robot across a $135^{\circ}$ elbow with outer radius of $70 \mathrm{~cm}$

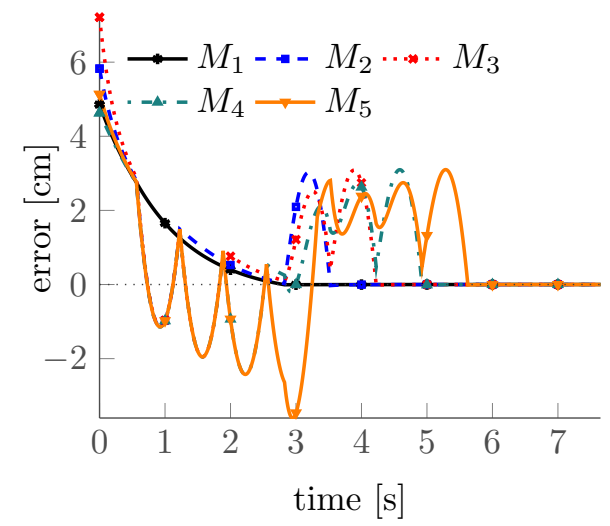

(a) tracking error

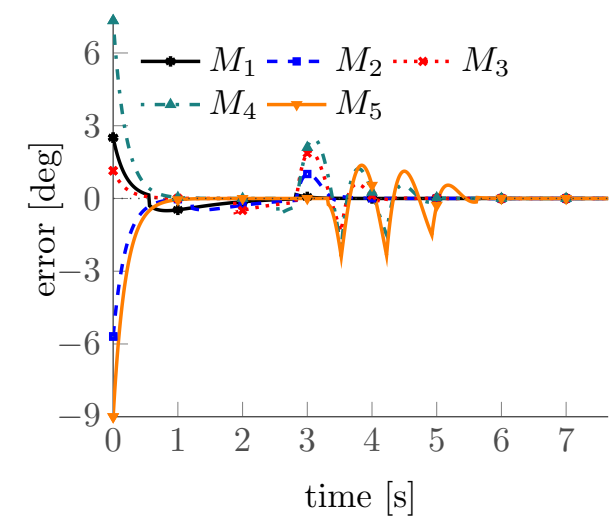

(b) orientation error

Figure 19: Position and orientation errors of a 5-module robot across a $135^{\circ}$ elbow with outer radius of $70 \mathrm{~cm}$

\section{Conclusions}

We have presented the kinematics of a planar snake-like robot. The robotic system is conceived to be deployed and autonomously operate in pipe-like environment, in order to perform tasks associated to inspection and exploration. The robot is an articulated multibody system comprised of identical 


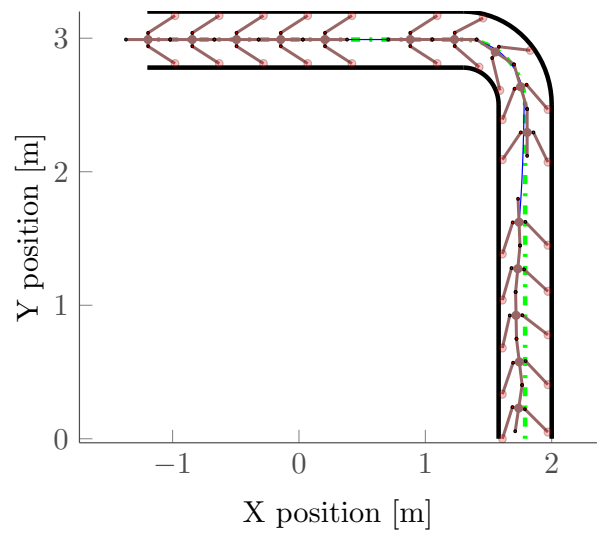

Figure 20: Snapshots of a 5-module robot across a $90^{\circ}$ elbow with outer radius of $70 \mathrm{~cm}$

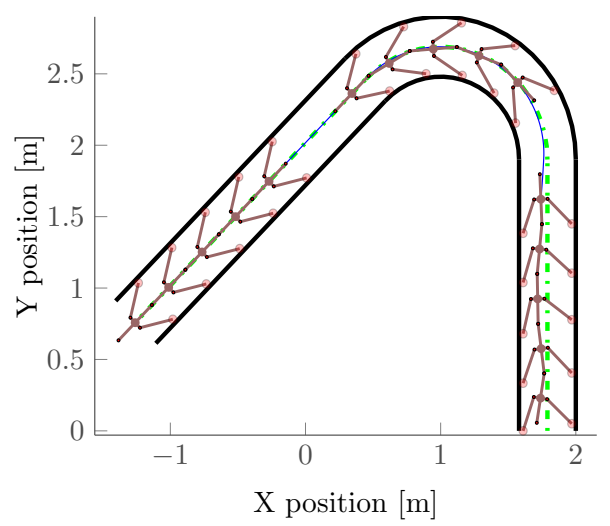

Figure 21: Snapshots of a 5-module robot across a $270^{\circ}$ elbow with outer radius of $1 \mathrm{~m}$

modules. Each module is modelled as a parallel manipulator (four bar mechanism) on a mobile base. Position and velocity kinematics were derived for a single module and for the full robot. Parallel and serial singularities for a single module were analytically characterized. Dimensional synthesis of the arms has been posed as an optimization problem to maximize a dexterity index, and the optimal arm length when crossing an elbow has been 


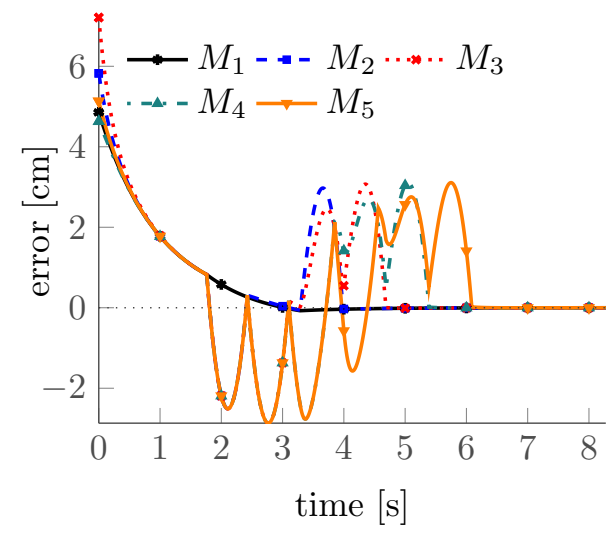

(a) tracking error

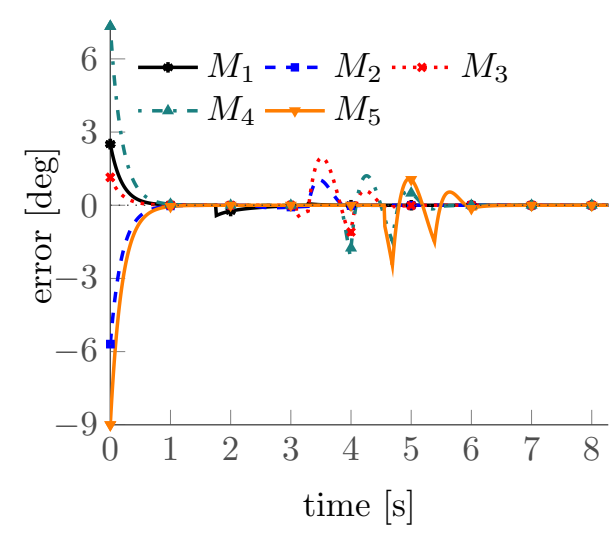

(b) orientation error

Figure 22: Position and orientation errors of a 5-module robot across a $90^{\circ}$ elbow with outer radius of $70 \mathrm{~cm}$

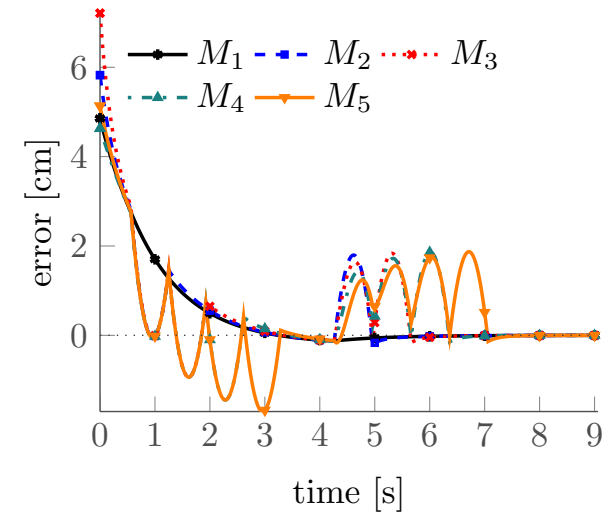

(a) tracking error

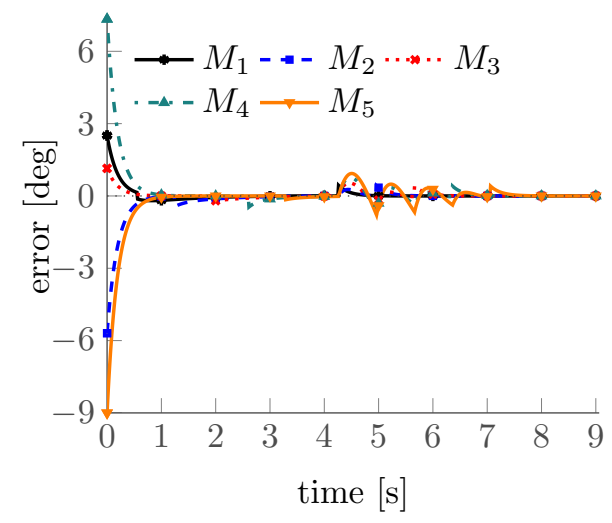

(b) orientation error

Figure 23: Position and orientation errors of a 5-module robot across a $270^{\circ}$ elbow with outer radius of $1 \mathrm{~m}$

determined.

The kinematics has been illustrated by motion control simulations of path following in a pipe-like environment. We applied the path following control scheme for autonomous navigation in a pipe with two scenarios of different 
complexities: a restriction of cross section in a straight pipe and an elbow with different angles. Simulations show that the structure presented in this work is suitable for navigation in pipes with varying radii, and critical manoeuvres that involve crossing elbows are kinematically admissible provided that the geometry of the robot is compatible with the environment. With respect to the $n$-trailer architecture, the kinematics proposed here is characterized by augmented degrees of freedom that can be crucial for autonomous operation purposes, as it allows to access more states of the articulated mechanism.

\section{References}

[1] K. Abdelnour, A. Stinchcombe, M. Porfiri, J. Zhang, and S. Childress. Wireless Powering of Ionic Polymer Metal Composites Toward Hovering Microswimmers. IEEE-ASME Transactions on Mechatronics, 17(5):924-935, Oct 2012.

[2] C. Altafini. A path tracking criterion for an LHD articulated vehicle. International Journal of Robotic Research, 18(5):435-441, 1999.

[3] C. Altafini. Some properties of the general n-trailer. International Journal of Control, 74(4):409-424, 2001.

[4] C. Altafini. Following a path of varying curvature as an output regulation problem. IEEE Transactions on Automatic Control, 47(9):1551- 
$1556,2002$.

[5] C. Altafini. Path following with reduced off-tracking for multibody wheeled vehicles. IEEE Transactions on Control Systems Technology, 11(4):598-605, 2003.

[6] J. Barraquand and J. Latombe. Nonholonomic multibody mobile robots: Controllability and motion planning in the presence of obstacles. Algorithmica, 10(2):121-155, 1993.

[7] Z. Y. Bayraktaroglu and P. Blazevic. Understanding snakelike locomotion through a novel push-point approach. Journal of Dynamic Systems, Measurement, and Control, 127(1):146-152, 2005.

[8] D. S. Bernstein. Matrix Mathematics: Theory, Facts, and Formulas (Second Edition). Princeton University Press, 2011.

[9] P. Bolzern, R. DeSantis, A. Locatelli, and S. Togno. Dynamic model of a two-trailer articulated vehicle subject to nonholonomic constraints. Robotica, 14:445-450, 1996.

[10] G. S. Chirikjian and J. W. Burdick. The kinematics of hypeer-redundant robot locomotion. IEEE Transactions on Robotics and Automation, 11(6):781-793, DEC 1995.

[11] J. E. Colgate and K. M. Lynch. Mechanics and control of swimming: A review. IEEE Journal of Oceanic Engineering, 29(3):660-673, July 2004. 
[12] P. I. Corke and P. R. Ridley. Steering kinematics for a center-articulated mobile robot. IEEE Transactions on Robotics, 17(2):215-218, 2001.

[13] C. C. de Wit, A. D. NDoudi-Likoho, and A. Micaelli. Nonlinear control for a train-like vehicle. The International Journal of Robotic Research, 16(3):300-319, 1997.

[14] S. A. Fjerdingen, P. Liljeback, and A. A. Transeth. A snake-like robot for internal inspection of complex pipe structures (PIKo). In Proceedings of the 2009 IEEE/RSJ International Conference on Intelligent Robots and Systems, 2009.

[15] F. Frenet. Sur les courbes à double courbure. Journal des mathématiques pures et appliquées, 17:437-447, 1852.

[16] E. F. Fukushima and S. Hirose. Attitude and Steering Control of the Long Articulated Body Mobile Robot KORYU. In H. Zhang, editor, Climbing and Walking Robots, Towards New Applications, chapter 2, pages 23-48. InTech, 2007.

[17] C. Gosselin and J. Angeles. Singularity analysis of closed-loop kinematic chains. IEEE Transactions on Robotics and Automation, 6(3):281-290, 1990.

[18] S. Hirose and A. Morishima. Design and control of a mobile robot with an articulated body. Journal of Robotics Research, 9(2):99-113, 1990. 
[19] M. Z. Huang. Design of a planar parallel robot for optimal workspace and dexterity. International Journal of Advanced Robotic Systems, 8(4):176$183,2011$.

[20] A. Jamoussi. Robotic NDE: A new solution for in-line pipe inspection. In Middle East Nondestructive Testing Conference and Exhibition, 2005.

[21] H. Kimura and S. Hirose. Development of Genbu : Active wheel passive joint articulated mobile robot. In IEEE International Conference on Intelligent Robots and Systems, pages 823-828, 2002.

[22] P. S. Krishnaprasad and D. P. Tsakiris. G-snakes: Nonholonomic kinematic chains on lie groups. In Proceedings of the IEEE Conference on Decision and Control, volume 3, pages 2955-2960, 1994.

[23] M. Lacagnina, S. Guccione, G. Muscato, and R. Sinatra. Modelling and simulation of multibody mobile robot for volcanic environment explorations. In IEEE/RSJ International Conference on Intelligent Robots and Systems, pages 823-828, 2002.

[24] P. Liljebäck, O. Stavdahl, and K. Y. Pettersen. Modular pneumatic snake robot: 3D modelling, implementation and control. Modeling, Identification and Control, 29(1):21-28, 2008.

[25] D. A. Lizarraga, P. Morin, and C. Samson. Chained form approximation of a driftless system. Application to the exponential stabilization of the 
general N-trailer system. International Journal of Control, 74(16):16121629, NOV 2001.

[26] S. Ma and N. Tadokoro. Analysis of creeping locomotion of a snake-like robot on a slope. Autonomous Robots, 20(1):15-23, 2006.

[27] S. Marras and M. Porfiri. Fish and robots swimming together: attraction towards the robot demands biomimetic locomotion. Journal of the Royal Society Interface, 9(73):1856-1868, Aug 2012.

[28] J. L. Martinez, J. Morales, A. Mandow, and A. J. Garcia-Cerezo. Steering limitations for a vehicle pulling passive trailers. IEEE Transactions on Control Systems technology, 16(4):809-818, 2008.

[29] J. P. Merlet. Parallel Robots. Springer, 2006.

[30] J.M. Mirats Tur and W. Garthwaite. Robotic devices for water main in-pipe inspection: A survey. Journal of Field Robotics, 27(4):491-508, 2010 .

[31] B. Murugendran, A. A. Transeth, and S. A. Fjerdingen. Modeling and Path-following for a Snake Robot with Active Wheels. In 2009 IEEERSJ International Conference on Intelligent Robots and Systems, pages 3643-3650, 2009.

[32] C. D. Onal, R. J. Wood, and D. Rus. An Origami-Inspired Approach to Worm Robots. IEEE-ASME Transactions on Mechatronics, 18(2):430438, APR 2013. 
[33] M. Ono and S. Kato. A study of an earthworm type inspection robot movable in long pipes. International Journal of Advanced Robotic Systems, 6(1):85-90, 2010.

[34] J. Ostrowski and J. Burdick. Gait kinematics for a serpentine robot. In Proceedings of the IEEE International Conference on Robotics and Automation, volume 2, pages 1294-1299, 1996.

[35] G. Poi, C. Scarabeo, and B. Allotta. Traveling wave locomotion hyperredundant mobile robot. In IEEE International Conference on Robotics and Automation, volume 1, pages 418-423, 1998.

[36] H. Schempf, E. Mutschler, V. Goltsberg, G. Skoptsov, A. Gavaert, and G. Vradis. Explorer: Untethered real-time gas main assessment robot system. In Proc. of Int Workshop on Advances in Service Robotics, 2003.

[37] H. Shin, K.-M. Jeong, and J.-J. Kwon. Development of a snake robot moving in a small diameter pipe. In Proceedings of the International Conference on Control, Automation and Systems, 2010.

[38] K. Suzumori, S. Wakimoto, and M. Takata. A miniature inspection robot negotiating pipes of widely varying diameter. In IEEE International Conference on Robotics and Automation, pages 2735-2740, 2003.

[39] J. L. Tangorra, S. N. Davidson, I. W. Hunter, P. G. A. Madden, G. V. Lauder, H. Dong, M. Bozkurttas, and R. Mittal. The development of a 
biologically inspired propulsor for unmanned underwater vehicles. IEEE Journal of Oceanic Engineering, 32(3):533-550, July 2007.

[40] A. A. Transeth, R. I. Leine, C. Glocker, and K. Y. Pettersen. 3-D snake robot motion: Nonsmooth modeling, simulations, and experiments. IEEE Transactions on Robotics, 24(2):361-376, Apr 2008.

[41] A. A. Transeth, R. I. Leine, C. Glocker, K. Y. Pettersen, and P. Liljebäck. Snake robot obstacle-aided locomotion: modeling, simulations, and experiments. IEEE Transactions on Robotics, 24(1):88-104, 2008.

[42] A. A. Transeth and K. Y. Pettersen. Developments in snake robot modeling and locomotion. In International Conference on Control, Automation, Robotics and Vision ICARCV, pages 1-8, 2006.

[43] A. A. Transeth, K. Y. Pettersen, and P. Liljebäck. A survey on snake robot modeling and locomotion. Robotica, 27(07):999-1015, 2009.

[44] P. Wiriyacharoensunthorn and S. Laowattana. Analysis and design of a multi-link mobile robot (serpentine). In IEEE International Conference on Industrial Technology, volume 2, pages 694-699, 2002.

[45] J. Yu, R. Ding, Q. Yang, M. Tan, W. Wang, and J. Zhang. On a Bio-inspired Amphibious Robot Capable of Multimodal Motion. IEEEASME Transactions on Mechatronics, 17(5):847-856, Oct 2012. 
[46] J. Yu, Z. Su, M. Wang, M. Tan, and J. Zhang. Control of Yaw and Pitch Maneuvers of a Multilink Dolphin Robot. IEEE Transactions on Robotics, 28(2):318-329, Apr 2012.

[47] J. Yuan, Y. Huang, Y. Kang, and Z. Liu. A strategy of path following control for multi-steering tractor-trailer mobile robot. In Proc. of the IEEE International Conference on Robotics and Biomimetics, 2004.

\section{List of Figures}

1 Schematic of a configuration of the multibody vehicle . . . . 5

2 The articulated multibody robot Explorer (source: http:// wWw.rec.ri.cmu.edu/projects/explorer/, active on July

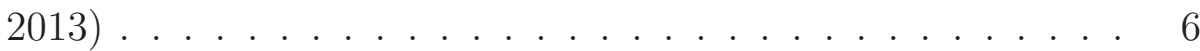

3 Sketch of a module with relevant parameter and kinematic descriptors . . . . . . . . . . . . . . . 8

4 Kinematic scheme of one module . . . . . . . . . . . 8

$5 \quad$ Illustration of the kinematic constraint describing the contact of the right wheel with the pipe wall . . . . . . . . 10

6 Two serial singularities: (a) both arms are normal to the pipe walls, (b) only one arm is normal to the wall . . . . . . . 16

7 Right wheel contact with the pipe wall . . . . . . . . . 17

8 Illustration of the module in a $135^{\circ}$ elbow . . . . . . . . . 18 
9 Dexterity index $\delta$ versus relative orientation $\theta-\theta_{\nu} \in[-\pi / 2, \pi / 4]$, for different arm lengths $l \ldots \ldots$. . . . . . . . . . 20

10 Average dexterity index over the full span $\left[\theta_{\nu}-\pi / 4, \theta_{\nu}+\pi / 4\right]$ of the global orientations of the module, for different values of $l 20$

11 Workspace illustration for orientation $\theta=\theta_{\nu}$. . . . . . . . . 21

12 Workspace illustration for $\theta=\theta_{\nu}-\frac{\pi}{6} \ldots$. . . . . . . . 21

13 Frenet frames for path following control . . . . . . . . . 23

14 Position and orientation errors in an elbow with one module . 25

15 Module's snapshots in an elbow ............. . 25

16 Snapshots of a 5-module robot in a pipe of varying diameter . 29

17 Position and orientation errors of a 5-module robot in a pipe of varying diameter . . . . . . . . . . . . . . . . . . . 29

18 Snapshots of a 5-module robot across a $135^{\circ}$ elbow with outer radius of $70 \mathrm{~cm} \ldots \ldots \ldots \ldots \ldots$

19 Position and orientation errors of a 5-module robot across a $135^{\circ}$ elbow with outer radius of $70 \mathrm{~cm}$. . . . . . . . 31

20 Snapshots of a 5-module robot across a $90^{\circ}$ elbow with outer radius of $70 \mathrm{~cm} \ldots \ldots \ldots \ldots . \ldots . \ldots . \ldots . \ldots 32$

21 Snapshots of a 5-module robot across a $270^{\circ}$ elbow with outer radius of $1 \mathrm{~m} \ldots \ldots \ldots . \ldots \ldots 32$

22 Position and orientation errors of a 5-module robot across a $90^{\circ}$ elbow with outer radius of $70 \mathrm{~cm} \ldots . . . . . . .33$ 
23 Position and orientation errors of a 5-module robot across a $270^{\circ}$ elbow with outer radius of $1 \mathrm{~m} \ldots . . . . . . .33$ 